ANNALES

POLONICI MATHEMATICI

LIV.1 (1991)

\title{
Remark on hyperbolic embeddability of relatively compact subspaces of complex spaces
}

\author{
by Do Duc ThaI (Hanoi)
}

\begin{abstract}
Abstruct. The characterization of hyperbolic embeddability of relatively compact subspaces of a complex space in terms of extension of holomorphic maps from the punctured disc and of limit complex lines is given.
\end{abstract}

We assume that complex spaces are connected and have a countable topology. Put $D=\{z \in C:|z|<1\}$ and $D^{*}=D \backslash\{0\}$.

Let $M$ be a subspace of a complex space $X$. We say that $M$ has the $D^{*}$-extension property for $X$ if every holomorphic map of $D^{*}$ into $M$ can be extended to a holomorphic map of $D$ into $X$.

Recall that $M$ is hyperbolically embedded in $X$ if for $x, y \in \bar{M}, x \neq y$, there exist open neighbourhoods $U$ of $x$ and $V$ of $y$ such that $d_{M}(U \cap M, V \cap M)>0$, where for each complex space $Z$ we denote by $d_{z}$ the Kobayashi semidistance on $Z$. It is known [2] that if $M$ is hyperbolically embedded in $X$ then $M$ has the $D^{*}$-extension property for $X$. Some criterions for hyperbolic embedding can be found in [3]. In a particular case when $M$ and $X$ are complex manifolds, $M$ is locally complete hyperbolic and relatively compact Zaidenberg has proved [4] that $M$ is hyperbolically embedded in $X$ if $M$ contains no complex lines and $\partial M$ contains no limit complex lines. Here by a limit complex line of $\partial M$ we mean a non-constant holomorphic map of $\mathbf{C}$ into $X$ which can be approximated on each $D_{r}=r D$ by holomorphic maps of $D_{r}$ into $M$. In case where $X$ is a complex space and $M$ is a complement of a hypersurface the result has been established in [3].

The aim of this note is to prove the following

THEOREM. Let $M$ be a locally complete hyperbolic and relatively compact subspace of a complex space $X$. Then the following are equivalent:

(i) $M$ is hyperbolically embedded in $X$.

(ii) $M$ has the $D^{*}$-extension property for $X$ and $\partial M$ contains no limit complex lines.

(iii) $M$ contains no complex lines and $\partial M$ contains no limit complex lines.

Moreover, if one of the above conditions holds, then $M$ is complete hyperbolic. 
Proof. It is known [3] that $M$ is hyperbolically embedded in $X$ if and only if

$$
c(M):=\sup \{\|d f(0)\|: f \in H(D, M)\}<\infty
$$

where $H(D, M)$ denotes the space of holomorphic maps from $D$ into $M$ equipped with the compact-open topology.

(i) $\Rightarrow$ (ii). By [2], $M$ has the $D^{*}$-extension property for $X$. Thus it remains to show that $\partial M$ contains no limit complex lines. This is an immediate consequence of the inequality $c(M)<\infty$.

(ii) $\Rightarrow$ (iii). It suffices to prove that $M$ contains no complex lines. Assume that there exists a non-constant holomorphic $\operatorname{map} f: C \rightarrow M$. Observe that the map

$$
g:(C \backslash\{0\}) \cup\{\infty\}=C P^{1} \ni z \mapsto \begin{cases}1 / z & \text { for } z \in \mathbf{C} \backslash\{0\}, \\ 0 & \text { for } z=\infty,\end{cases}
$$

is holomorphic.

By (ii), the map $(f \circ g) \mid D^{*}$ can be extended to a holomorphic map on $D$. Hence $f \circ g$ can be extended to a holomorphic map $0: \mathbf{C} P^{1} \rightarrow X$. Since $f \circ g \neq$ const, it follows that 0 is finite. Take a holomorphic map $\sigma: D^{*} \rightarrow \mathbf{C} \subset \mathbf{C} P^{1}$ which cannot be extended to a holomorphic map of $D$ into $C P^{1}$. By hypothesis $\beta=\theta \sigma$ can be extended to a holomorphic map $\beta: D \rightarrow X$. Since $\theta$ is finite, there exists a neighbourhood $U$ of $\beta(0)$ such that $0^{-1}(U)$ is isomorphic to a bounded domain in $\mathbf{C}$. Thus for sufficiently small $\varepsilon>0, \sigma \mid D_{\varepsilon}^{*}$ can be extended to a holomorphic map of $D_{t}$ into $0^{-1}(U)$. This is impossible.

(iii) $\Rightarrow$ (i). It suffices to show that $c(M)<\infty$. Assume that $c(M)=\infty$. Then there exists a sequence $\left\{f_{n}\right\} \subset H(D, M)$ such that $\left\|d f_{n}(0)\right\|=r_{n} \rightarrow \infty$. For each $n \geqslant 1$ consider the map $g_{n}: D_{r_{n}} \rightarrow M, z \mapsto \int_{n}\left(z / r_{n}\right)$. Then $\left\|d g_{n}(0)\right\|=1$. As in [3] there exists a sequence of holomorphic maps $\varphi_{n}: D_{r_{n}} \rightarrow M$ which is uniformly convergent on every compact subset of every fixed disc in $\mathbf{C}$ to a holomorphic map $\varphi: \mathbf{C} \rightarrow X$ and $\left\|d \varphi_{n}(0)\right\|=1$ for every $n \geqslant 1$. Clearly $\varphi$ is not constant since $\|d \varphi(0)\|=\lim \left\|d \varphi_{n}(0)\right\|=1$. Since $\partial M$ contains no limit complex lines, $\varphi(\mathbf{C}) \cap M \neq \varnothing$. Put

$$
\begin{gathered}
Z=\{z \in C: \text { there exists an open neighbourhood } U \\
\text { of } z \text { such that } \varphi(U) \subset \partial M\} .
\end{gathered}
$$

Assume that $Z \neq \varnothing$. Let $z_{0} \in \partial Z$. Take an open neighbourhood $U$ of $\varphi\left(z_{0}\right)$ such that $U \cap M$ is complete hyperbolic. Since $\left\{\varphi_{n}\right\}$ uniformly converges to $\varphi$ on every compact set in $\mathrm{C}$, there exists an open neighbourhood $W$ of $z_{0}$ such that $\varphi_{n}(W) \subset U \cap M$ for $n \geqslant l$ and $\varphi(W) \subset U \cap \bar{M}$. From the complete hyperbolicity of $U \cap M$, it follows that $H(W, U \cap M)$ is normal [3].

Assume that the sequence $\left\{\varphi_{n} \mid W\right\}$ contains a subsequence which is compactly divergent. Without loss of generality we maly assume that the 
sequence $\left\{\varphi_{n} \mid W\right\}$ is compactly divergent. Choose $z_{1} \in W$ such that $\varphi\left(z_{1}\right)$ $\in U \cap M$. Then for every compact neighbourhood $K^{\prime}$ of $\varphi\left(z_{1}\right)$ in $U \cap M$, there exists $j$ such that $\varphi_{n}\left(z_{1}\right) \notin K^{\prime} \forall n \geqslant j$. Hence $\varphi_{n}\left(z_{1}\right) \rightarrow \varphi\left(z_{1}\right)$. This is a contradiction.

Thus we may assume that $\left\{\varphi_{n} \mid W\right\}$ converges to a holomorphic map in $H(W, U \cap M)$. Hence $\lim \varphi_{n}\left(z_{0}\right) \in U \cap M$. Thus $\varphi\left(z_{0}\right) \in U \cap M$, i.e. $\varphi\left(z_{0}\right) \notin \partial M$. This is a contradiction. Therefore $Z=\varnothing$.

Since $M$ contains no complex lines, $\varphi(C) \cap \partial M \neq \varnothing$. Let $z_{0} \in \mathbf{C}$ such that $\varphi\left(z_{0}\right) \in \partial M$. Reasoning as above, there exists an open neighbourhood $U$ of $\varphi\left(z_{0}\right)$ such that $U \cap M$ is complete hyperbolic and an open neighbourhood $W$ of $z_{0}$ such that $\varphi_{n}(W) \subset U \cap M \forall n \geqslant l$ and $\varphi(W) \subset U \cap \bar{M}$. By $Z=\varnothing$ there exists $z_{1} \in W$ such that $\varphi\left(z_{1}\right) \in U \cap M$. Reasoning as above, we may assume that the sequence $\left\{\varphi_{n} \mid W\right\}$ is convergent in $H(W, U \cap M)$. This, as above, leads to a contradiction. Hence $c(M)$ is finite and the implication (iii) $\Rightarrow$ (i) is proved.

Finally, by [3] if $M$ is as in the theorem and $M$ is hyperbolically embedded in $X$, then $M$ is complete hyperbolic.

The following is an immediate consequence of the above theorem.

COROLlary. Let $M$ be a compact complex space. Then the following are equivalent:

(i) $M$ is hyperbolic.

(ii) $M$ has the $D^{*}$-extension property for $M$.

(iii) $M$ contains no complex lines.

\section{References}

[1] R. Brody, Compact manifolds and hyperbolicity, Trans. Amer. Math. Soc. 235 (1978), 213-219.

[2] S. Kobayashi, Hyperbolic Manifolds and Holomorphic Mappings, Dekker, 1970.

[3] S. Lang, Introduction to Complex Hyperbolic Spaces, Springer-Verlag, 1987.

[4] M. Zaidenberg. Picard theorem and hyperbolicity, Siberian Math. J. 24 (6) (1983), 44-55.

DEPARTMENT OF MATHEMATICS, PEDAgogical INSTITUTE I OF haNOI

Honoi, Vioinam

Refu par la Rédaction le 05.11.1988

Révisé le 03.08.1989 